\title{
Quantifying concordant genetic effects of de novo mutations on multiple disorders
}

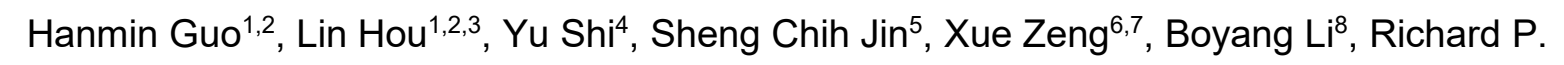
Lifton $^{6,7}$, Martina Brueckner ${ }^{6,9}$, Hongyu Zhao ${ }^{6,8,10, \#}$, Qiongshi Lu ${ }^{11, \#, *}$

${ }^{1}$ Center for Statistical Science, Tsinghua University, Beijing, China

${ }^{2}$ Department of Industrial Engineering, Tsinghua University, Beijing, China

${ }^{3}$ MOE Key Laboratory of Bioinformatics, School of Life Sciences, Tsinghua University, Beijing, China

${ }^{4}$ Yale School of Management, Yale University, New Haven, CT, USA

${ }^{5}$ Department of Genetics, Washington University School of Medicine, St. Louis, MO, USA

${ }^{6}$ Department of Genetics, Yale University, New Haven, CT, USA

${ }^{7}$ Laboratory of Human Genetics and Genomics, Rockefeller University, New York, USA

${ }^{8}$ Department of Biostatistics, Yale School of Public Health, New Haven, CT, USA

${ }^{9}$ Department of Pediatrics, Yale University School of Medicine, New Haven, CT, USA

10 Program of Computational Biology and Bioinformatics, Yale University, New Haven, CT, USA

${ }^{11}$ Department of Biostatistics and Medical Informatics, University of Wisconsin-Madison, Madison, WI, USA

\# These authors should be considered shared last author

* Correspondence:

Dr. Qiongshi Lu

Department of Biostatistics and Medical Informatics

University of Wisconsin-Madison

425 Henry Mall

Madison, WI, USA 53706

qlu@biostat.wisc.edu 


\section{Abstract}

Exome sequencing on tens of thousands of parent-proband trios has identified numerous deleterious de novo mutations (DNMs) and implicated risk genes for many disorders. Recent studies have suggested shared genes and pathways are enriched for DNMs across multiple disorders. However, existing analytic strategies only focus on genes that reach statistical significance for multiple disorders and require large trio samples in each study. As a result, these methods are not able to characterize the full landscape of genetic sharing due to polygenicity and incomplete penetrance. In this work, we introduce EncoreDNM, a novel statistical framework to quantify shared genetic effects between two disorders characterized by concordant enrichment of DNMs in the exome. EncoreDNM makes use of exome-wide, summary-level DNM data, including genes that do not reach statistical significance in single-disorder analysis, to evaluate the overall and annotation-partitioned genetic sharing between two disorders. Applying EncoreDNM to DNM data of nine disorders, we identified abundant pairwise enrichment correlations, especially in genes intolerant to pathogenic mutations and genes highly expressed in fetal tissues. These results suggest that EncoreDNM improves current analytic approaches and may have broad applications in DNM studies. 


\section{Introduction}

51

De novo mutations (DNMs) can be highly deleterious and provide important insights into the genetic cause for disease ${ }^{1}$. As the cost of sequencing continues to drop, whole-exome sequencing (WES) studies conducted on tens of thousands of family trios have pinpointed numerous risk genes for a variety of disorders ${ }^{2-4}$. In addition, accumulating evidence suggests that risk genes enriched for pathogenic DNMs may be shared by multiple disorders ${ }^{5-9}$. These shared genes could reveal biological pathways that play prominent roles in disease etiology and shed light on clinically heterogeneous yet genetically related diseases ${ }^{7-9}$.

Most efforts to identify shared risk genes directly compare genes that are significantly associated with each disorder ${ }^{10,11}$. There have been some successes with this approach in identifying shared genes and pathways (e.g., chromatin modifiers) underlying developmental disorder (DD), autism spectrum disorder (ASD), and congenital heart disease (CHD), thanks to the large trio samples in these studies $3,4,12$, whereas findings in smaller studies remain suggestive ${ }^{13,14}$. Even in the largest studies to date, statistical power remains moderate for risk genes with weaker effects ${ }^{3,15}$. It is estimated that more than 1,000 genes associated with DD remain undetected ${ }^{3}$. Therefore, analytic approaches that only account for top significant genes cannot capture the full landscape of genetic sharing in multiple disorders. Recently, a Bayesian framework was proposed to jointly analyze DNM data of two diseases and improve risk gene mapping ${ }^{9}$. Although some parameters in this framework can quantify shared genetics between diseases, the statistical property of these parameter estimates have not been studied. There is a pressing need for powerful, robust, and interpretable methods that quantify concordant DNM association patterns for multiple disorders using exome-wide DNM counts.

Recent advances in estimating genetic correlations using summary data from genome-wide association studies (GWAS) may provide a blueprint for approaching this problem in DNM research ${ }^{16}$. Modeling "omnigenic" associations as independent random effects, linear mixedeffects models leverage genome-wide association profiles to quantify the correlation between additive genetic components of multiple complex traits ${ }^{17-20}$. These methods have identified ubiquitous genetic correlations across many human traits and revealed significant and robust genetic correlations that could not be inferred from significant GWAS associations alone ${ }^{21-24}$.

Here, we introduce EncoreDNM (Enrichment correlation estimator for De Novo Mutations), a novel statistical framework that leverages exome-wide DNM counts, including genes that do not reach exome-wide statistical significance in single-disorder analysis, to estimate concordant DNM associations between disorders. EncoreDNM uses a generalized linear mixed-effects model to quantify the occurrence of DNMs while accounting for de novo mutability of each gene and technical inconsistencies between studies. We demonstrate the performance of EncoreDNM through extensive simulations and analyses of DNM data of nine disorders. 


\section{Results}

91

\section{Method overview}

DNM counts in the exome deviate from the null (i.e., expected counts based on de novo mutability) when mutations play a role in disease etiology. Disease risk genes will show enrichment for deleterious DNMs in probands and non-risk genes may be slightly depleted for DNM counts. Our goal is to estimate the correlation of such deviation between two disorders, which we refer to as the DNM enrichment correlation. More specifically, we use a pair of mixed-effects Poisson regression models to quantify the occurrence of DNMs in two studies.

$$
\begin{gathered}
{\left[\begin{array}{l}
Y_{i 1} \\
Y_{i 2}
\end{array}\right] \sim \operatorname{Poisson}\left(\left[\begin{array}{l}
\lambda_{i 1} \\
\lambda_{i 2}
\end{array}\right]\right),} \\
\log \left(\left[\begin{array}{l}
\lambda_{i 1} \\
\lambda_{i 2}
\end{array}\right]\right)=\left[\begin{array}{l}
\beta_{1} \\
\beta_{2}
\end{array}\right]+\log \left(\left[\begin{array}{l}
2 N_{1} m_{i} \\
2 N_{2} m_{i}
\end{array}\right]\right)+\left[\begin{array}{l}
\phi_{i 1} \\
\phi_{i 2}
\end{array}\right], \\
{\left[\begin{array}{l}
\phi_{i 1} \\
\phi_{i 2}
\end{array}\right] \sim \operatorname{MVN}\left(\left[\begin{array}{l}
0 \\
0
\end{array}\right],\left[\begin{array}{cc}
\sigma_{1}^{2} & \rho \sigma_{1} \sigma_{2} \\
\rho \sigma_{1} \sigma_{2} & \sigma_{2}^{2}
\end{array}\right]\right) .}
\end{gathered}
$$

Here, $Y_{i 1}, Y_{i 2}$ are the DNM counts for the $i$-th gene and $N_{1}, N_{2}$ are the number of parent-proband trios in two studies, respectively. The log Poisson rates of DNM occurrence are decomposed into three components: the elevation component, the background component, and the deviation component. The elevation component $\beta_{k}(k=1,2)$ is a fixed effect term adjusting for systematic, exome-wide bias in DNM counts. One example of such bias is the batch effect caused by different sequencing and variant calling pipelines in two studies. The elevation parameter $\beta_{k}$ tends to be larger when DNMs are over-called with higher sensitivity and smaller when DNMs are undercalled with higher specificity ${ }^{25}$. The background component $\log \left(2 N_{k} m_{i}\right)$ is a gene-specific fixed effect that reflects the expected mutation counts determined by the genomic sequence context under the null ${ }^{26} . m_{i}$ is the de novo mutability for the $i$-th gene, and $2 N_{1} m_{i}$ and $2 N_{2} m_{i}$ are the expected DNM counts in the $i$-th gene under the null in two studies. The deviation component $\phi_{i k}$ is a gene-specific random effect that quantifies the deviation of DNM profile from what is expected under the null. $\phi_{i 1}$ and $\phi_{i 2}$ follow a multivariate normal distribution with dispersion parameters $\sigma_{1}$ and $\sigma_{2}$ and a correlation $\rho$. DNM enrichment correlation is denoted by $\rho$ and is our main parameter of interest. It quantifies the concordance of DNM burden in two disorders.

Parameters in this model can be estimated using a Monte Carlo maximum likelihood estimation (MLE) procedure. Standard errors of the estimates are obtained through inversion of the observed Fisher information matrix. In practice, we use annotated DNM data as input and fit mixed-effects Poisson models for each variant class separately: loss of function (LoF), deleterious missense (Dmis, defined as MetaSVM-deleterious), tolerable missense (Tmis, defined as MetaSVMtolerable), and synonymous (Figure 1). More details about model setup and parameter estimation are discussed in Methods. 

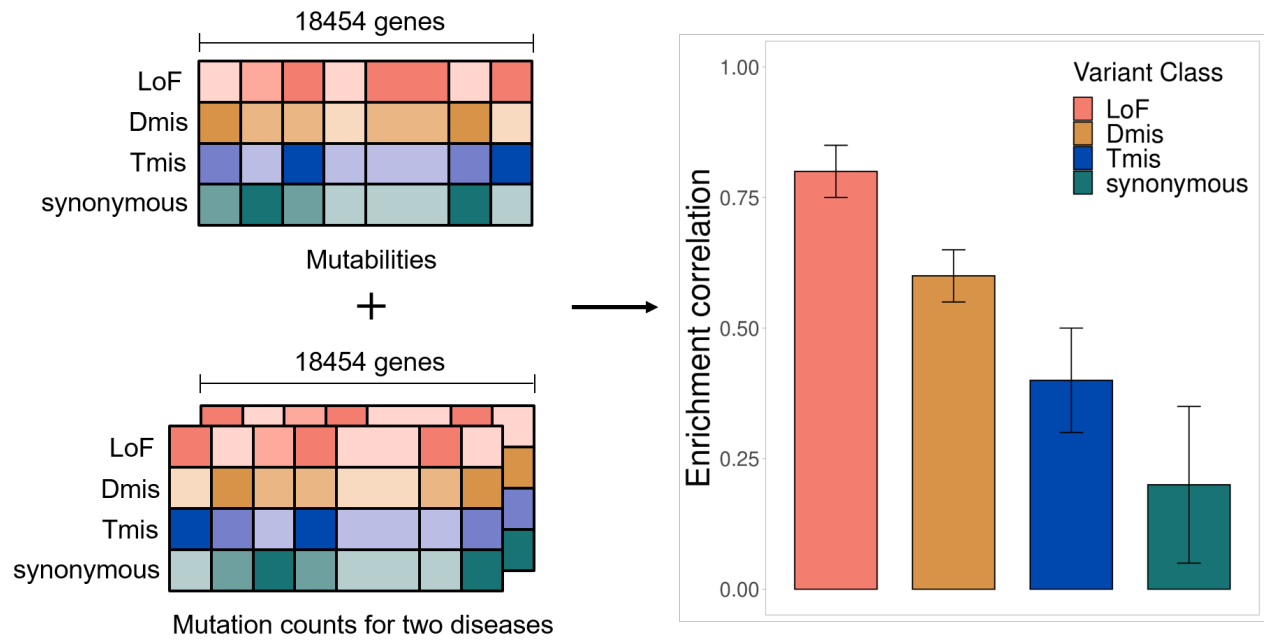

Figure 1. EncoreDNM workflow. The inputs of EncoreDNM are de novo mutability of each gene and exome-wide, annotated DNM counts from two studies. We fit a mixed-effects Poisson model to estimate the DNM enrichment correlation between two disorders for each variant class.

\section{Simulation results}

We conducted simulations to assess the parameter estimation performance of EncoreDNM in various settings. We focused on two variant classes, i.e., Tmis and LoF variants, since they have the highest and lowest median mutabilities in the exome. We used EncoreDNM to estimate the elevation parameter $\beta$, dispersion parameter $\sigma$, and enrichment correlation $\rho$ (Methods). Under various parameter settings, EncoreDNM always provided unbiased estimation of the parameters (Figure 2 and Supplementary Figures 1-2). Furthermore, the 95\% Wald confidence intervals achieved coverage rates close to $95 \%$ under all simulation settings, demonstrating the effectiveness of EncoreDNM to provide accurate statistical inference.

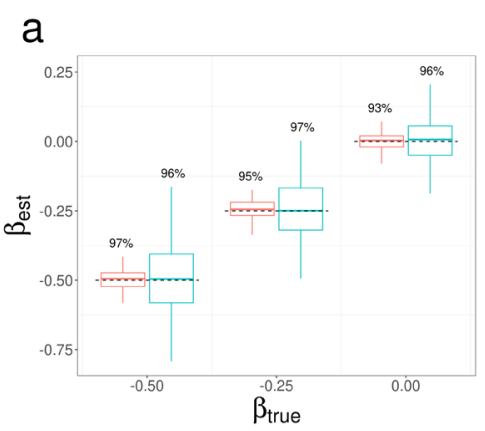

b

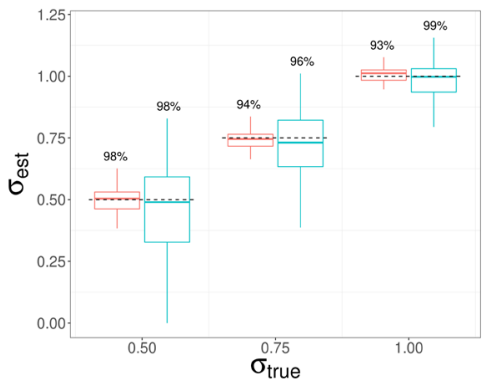

C

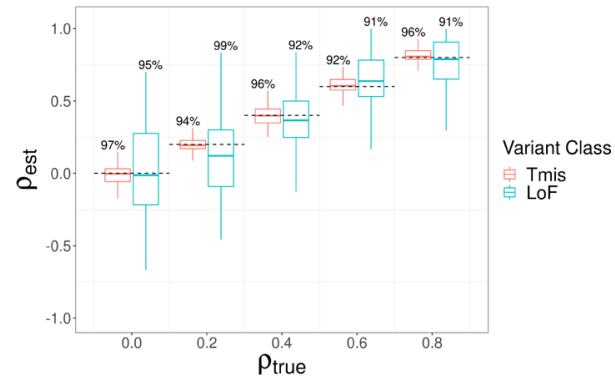

Figure 2. Parameter estimation results of EncoreDNM. (a) Boxplot of $\beta$ estimates in single-trait analysis with $\sigma$ fixed at 0.75. (b) Boxplot of $\sigma$ estimates in single-trait analysis with $\beta$ fixed at -0.25 . (c) Boxplot of $\rho$ estimates in cross-trait analysis with $\beta$ and $\sigma$ fixed at -0.25 and 0.75 . True parameter values are marked by dashed lines. The number above each box represents the coverage rate of $95 \%$ Wald confidence intervals. Each simulation setting was repeated 100 times.

Next, we compared the performance of EncoreDNM with $\operatorname{mTADA}^{9}$, a Bayesian framework that could estimate the proportion of shared risk genes for two disorders. First, we simulated DNM data under the mixed-effects Poisson model. We evaluated two methods across a range of 

produced false positive findings when the observed DNM counts were relatively small (e.g., due assessed the statistical power of two approaches under a baseline setting where type-I errors for both methods were controlled. As enrichment correlation increased, EncoreDNM achieved universally greater statistical power compared to mTADA (Figure $\mathbf{3 b}$ ).
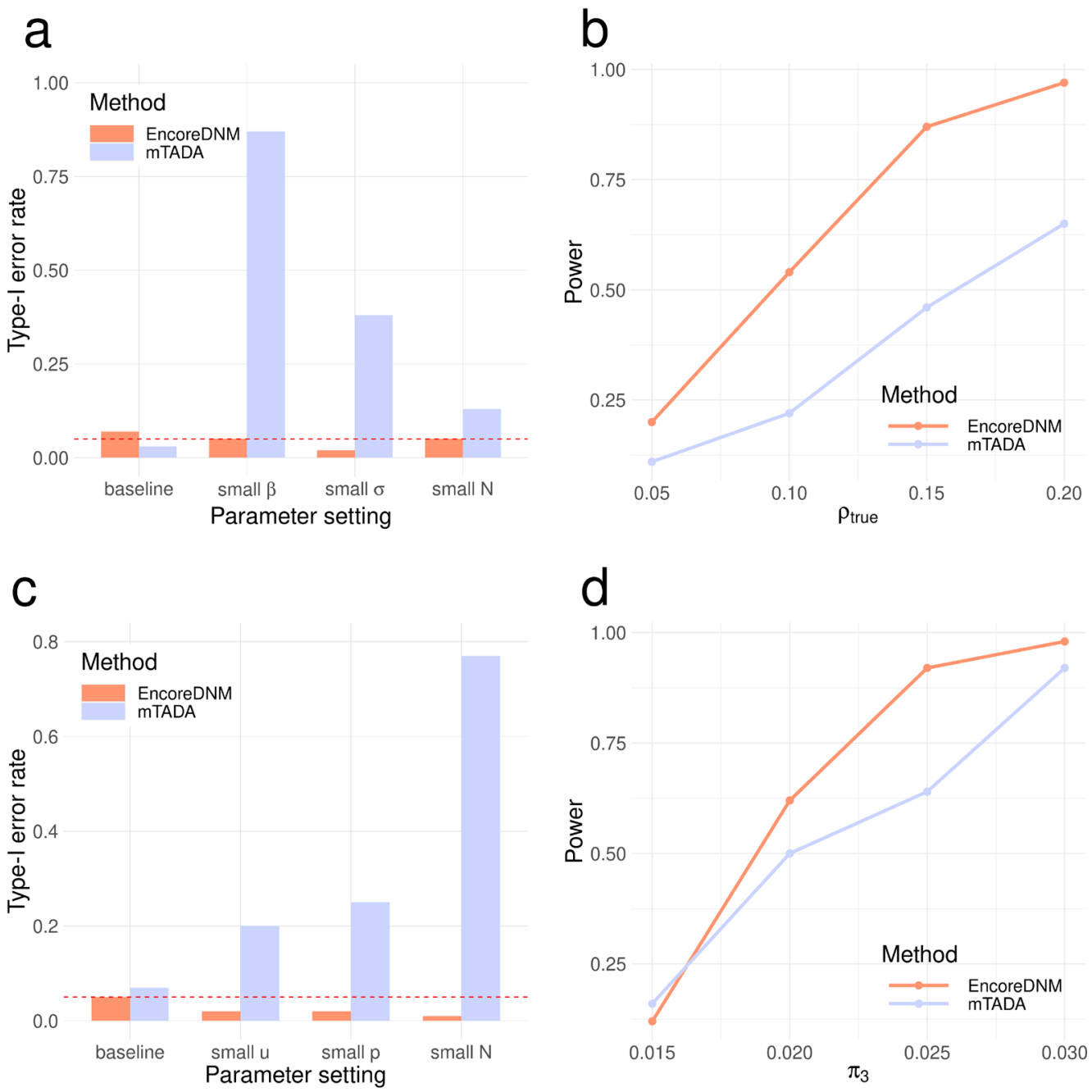

Figure 3. Comparison of EncoreDNM and mTADA. (a) Type-I error rates under a mixed-effects Poisson regression model. $(\beta, \sigma, N)$ were fixed at $(-0.25,0.75,5000)$ under the baseline setting, $(-1,0.75,5000)$ under a setting with small $\beta,(-0.25,0.5,5000)$ under a setting with small $\sigma$, and $(-0.25,0.75,1000)$ under a setting with small $\mathrm{N}$ for two disorders. (b) Statistical power of two methods under a mixed-effects Poisson regression model as the enrichment correlation increases. Parameters $(\beta, \sigma, N)$ were fixed at $(-0.25,0.75,5000)$ for both disorders. (c) Type-I error rates under a multinomial model. $\left(u, p, N, \pi^{S}\right)$ were fixed at $(0.95,0.25,5000,0.1)$ under the baseline setting, $(0.75,0.25,5000,0.1)$ under a setting with small $u$ (i.e., reduced total DNM counts), $(0.95,0.15,5000,0.1)$ under a setting with small $p$ (i.e., fewer probands explained by DNMs), and $(0.95,0.25,1000,0.1)$ under a setting with lower sample size. (d) Statistical power under a multinomial model with varying proportion of shared causal genes. Parameters $\left(u, p, N, \pi^{S}\right)$ were fixed at $(0.95,0.25,5000,0.1)$ for both disorders. Each simulation setting was repeated 100 times. 
To ensure a fair comparison, we also considered a mis-specified model setting where we randomly distributed the total DNM counts for each disorder into all genes with an enrichment in causal genes (Methods). EncoreDNM showed well-controlled type-I error across all simulation settings, whereas severe type-I error inflation arose for mTADA when the total mutation count, the proportion of probands that can be explained by DNMs, or the sample size were small (Figure 3c). Furthermore, we compared the statistical power of two methods under this model in a baseline setting where type-I error was controlled. EncoreDNM showed higher statistical power compared to mTADA as the fraction of shared causal genes increased (Figure 3d).

\section{Pervasive enrichment correlation of damaging DNMs among developmental disorders}

We applied EncoreDNM to DNM data of nine disorders (Supplementary Table 1; Methods): DD $(n=31,058 \text {; number of trios })^{3}, \operatorname{ASD}(n=6,430)^{4}$, schizophrenia $(S C Z ; n=2,772)^{15}$, CHD $(n=2,645)^{12}$, intellectual disability (ID; $n=820)^{2}$, Tourette disorder $(T D ; n=484)^{27}$, epileptic encephalopathies (EP; $\mathrm{n}=264)^{13}$, cerebral palsy $(\mathrm{CP} ; \mathrm{n}=250)^{14}$, and congenital hydrocephalus $(\mathrm{CH} ; \mathrm{n}=232)^{28}$. In addition, we also included 1,789 trios comprising healthy parents and unaffected siblings of ASD probands as controls ${ }^{29}$.

a

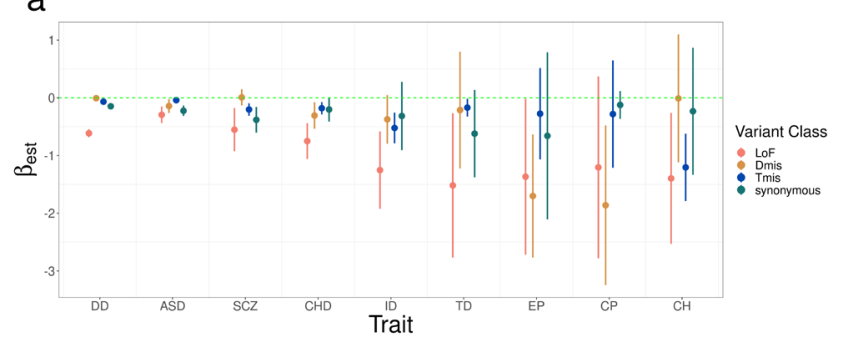

b

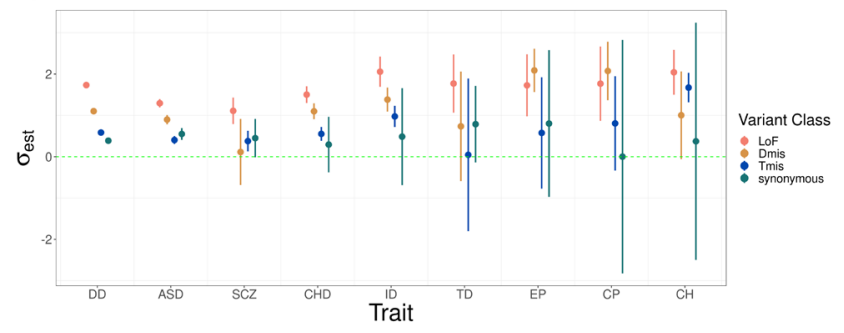

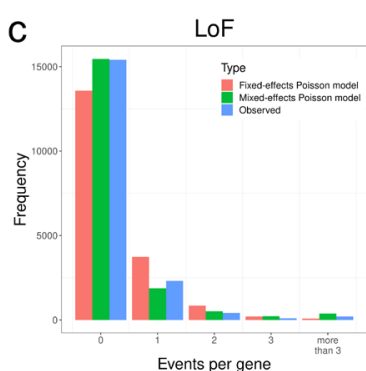

e

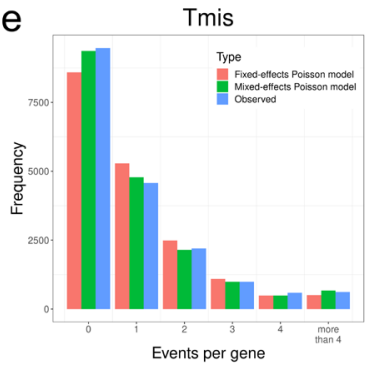

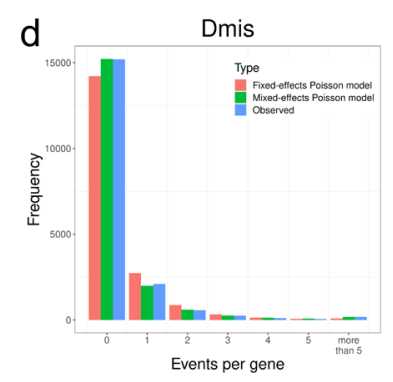

f

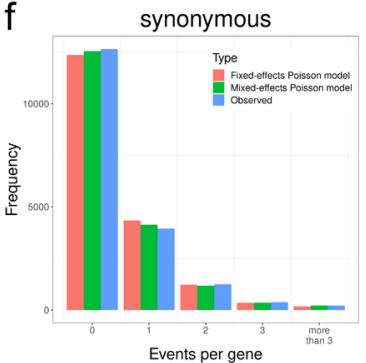

Figure 4. Model fitting results for nine disorders. (a, b) Estimation results of $\beta$ and $\sigma$ for nine disorders and four variant classes. Error bars represent 1.96*standard errors. (c-f) Distribution of DNM events per gene in four variant classes for DD. Red and green bars represent the expected frequency of genes under the fixed-effects and mixedeffects Poisson regression models, respectively. Blue bars represent the observed frequency of genes.

We first performed single-trait analysis for each disorder. The estimated elevation parameters (i.e., $\beta$ ) were negative for almost all disorders and variant classes (Figure 4a), with LoF variants showing particularly lower parameter estimates. This may be explained by more stringent quality control in LoF variant calling ${ }^{12}$ and potential survival bias ${ }^{30}$. It is also consistent with a depletion of LoF DNMs in healthy control trios ${ }^{7}$. The dispersion parameter estimates (i.e., $\sigma$ ) were higher 
for LoF variants than other variant classes (Figure 4b), which is consistent with our expectation that LoF variants have stronger effects on disease risk and should show a larger deviation from the null mutation rate in disease probands. We compared the goodness of fit of our proposed mixed-effects Poisson model to a simpler fixed-effects model without the deviation component (Methods). The expected distribution of recurrent DNM counts showed substantial and statistically significant improvement under the mixed-effects Poisson model (Figures 4c-f and

\section{Supplementary Figure 3).}

Next, we estimated pairwise DNM enrichment correlations for 9 disorders. In total, we identified (Figure 5 and Supplementary Figure 4), including 12 significant correlations for LoF variants, 7 for Dmis variants, 5 for Tmis variants, and only 1 significant correlation for synonymous variants. Notably, all significant correlations are positive (Supplementary Table 2). No significant correlation was identified between any disorder and healthy controls (Supplementary Figure 5). The number of identified significant correlations for each disorder was proportional to the sample size in each study (Spearman correlation $=0.70$ ) with controls being a notable outlier (Supplementary Figure 6).

a

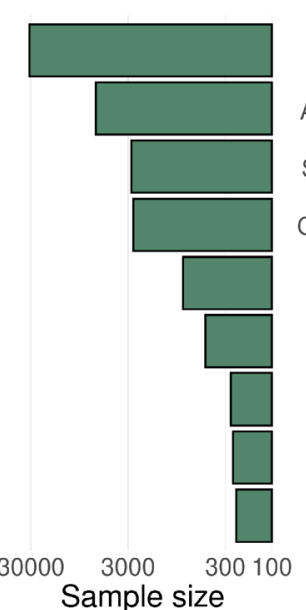

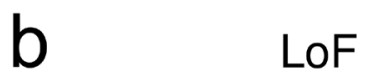

DD ASD SCZ CHD ID TD EP CP CH

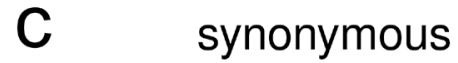

DD ASD SCZCHD ID TD EP CP CH

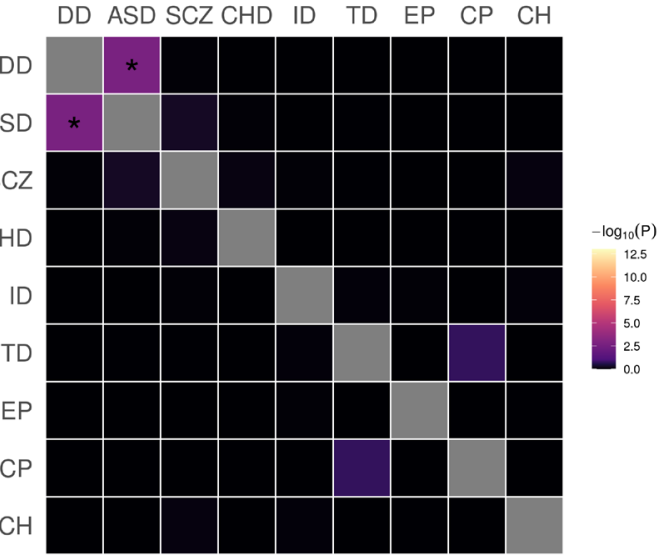

\section{0}

Figure 5. EncoreDNM identifies pervasive enrichment correlations of damaging DNMs among nine disorders. (a) shows sample size (i.e., number of trios) for each disease. X-axis denotes sample size on the log scale. (b, c) Heatmap of enrichment correlations for LoF and synonymous DNMs among nine disorders. Significant correlations $($ FDR $<0.05)$ are marked by asterisks. Results with $-\log _{10} P>13$ are truncated to 13 for visualization purpose.

We identified highly concordant and significant LoF DNM enrichment among DD, ASD, ID, and CHD, which is consistent with previous reports ${ }^{8-10,31}$. SCZ shows highly significant LoF correlations with DD and ID ( $p=2.0 e-3$ and $3.7 e-5)$, hints at a correlation with ASD $(p=0.012)$, but does not correlate strongly with $\mathrm{CHD}$. The positive enrichment correlation between ASD and CP in LoF variants $(\rho=0.81, \mathrm{p}=3.3 \mathrm{e}-3)$ is consistent with their co-occurrence ${ }^{32}$. The high enrichment correlation between ID and CP in LoF variants $(\rho=0.68, \mathrm{p}=1.0 \mathrm{e}-4)$ is consistent with the 
associations between ID and motor or non-motor abnormalities caused by $\mathrm{CP}^{33}$. A previous study also suggested significant genetic sharing of ID and CP by overlapping genes harboring rare damaging variants ${ }^{14}$. Here, we obtained consistent results after accounting for de novo mutabilities and potential confounding bias.

Some significant correlations identified in our analysis are consistent with phenotypic associations in epidemiological studies, but have not been reported using genetic data to the extent of our knowledge. For example, the LoF enrichment correlation between $\mathrm{CHD}$ and $\mathrm{CP}(\rho=0.88, \mathrm{p}=1.7 \mathrm{e}-$ 3 ) is consistent with findings that reduced supply of oxygenated blood in fetal brain due to cardiac malformations may be a risk factor for $\mathrm{CP}^{34}$. The enrichment correlation between ID and $\mathrm{CH}$ in LoF variants $(\rho=0.63, p=2.4 \mathrm{e}-3)$ is consistent with lower intellectual performance in a proportion of children with $\mathrm{CH}^{35}$.

Genes showing pathogenic DNMs in multiple disorders may shed light on the mechanisms underlying enrichment correlations (Supplementary Table 3). We identified five genes (i.e. CTNNB1, NBEA, POGZ, SPRED2, and KMT2C) with LoF DNMs in five different disorders and 21 genes had LoF DNMs in four disorders (Supplementary Table 4). These 26 genes with LoF variants in at least four disorders were significantly enriched for 63 gene ontology (GO) terms with FDR $<0.05$ (Supplementary Table 5). Chromatin organization $(p=7.8 \mathrm{e}-11)$, nucleoplasm $(p=2.8 \mathrm{e}-$ $10)$, chromosome organization $(p=6.8 e-10)$, histone methyltransferase complex $(p=1.4 e-9)$, and positive regulation of gene expression $(p=2.2 e-9)$ were the most significantly enriched GO terms. One notable example consistently included in these gene sets is CTNNB1 (Supplementary Figure 7). It encodes $\beta$-catenin, is one of the only two genes reaching genome-wide significance in a recent WES study for $\mathrm{CP}^{14}$, and also harbors multiple LoF variants in DD, ID, ASD, and CHD. It is a fundamental component of the canonical Wnt signaling pathway which is known to confer genetic risk for $A S D^{36}$. We also identified 157 recurrent LoF mutations in 45 genes (Supplementary Table 6). Most of these recurrent mutations were identified in DD due to its large sample size, but one mutation was identified in joint comparison of other disorders. FBXO11, encoding the F-box only protein 31, shows two recurrent p.Ser831fs LoF variants in ASD and $\mathrm{CH}$ (Supplementary Figure 8; $p=1.9 \mathrm{e}-3$; Methods). The F-box protein constitutes a substraterecognition component of the SCF (SKP1-cullin-F-box) complex, an E3-ubiquitin ligase complex responsible for ubiquitination and proteasomal degradation ${ }^{37}$. DNMs in FBXO11 have been previously implicated in severe ID individuals with autistic behavior problem ${ }^{38}$ and neurodevelopmental disorder ${ }^{39}$.

For comparison, we also applied mTADA to the same nine disorders and control trios. In total, mTADA identified 117 disorder pairs with significant genetic sharings at an FDR cutoff of 0.05 (Supplementary Table 7 and Supplementary Figure 9). Notably, we identified significant synonymous DNM correlations for all 36 disorder pairs and between all disorders and healthy

271 controls (Supplementary Figure 10). These results are consistent with the simulation results and suggest a substantially inflated false positive rate in mTADA. 
Further, we applied cross-trait linkage disequilibrium (LD) score regression ${ }^{18}$ to five of the nine disorders with publicly available GWAS summary statistics (Supplementary Table 8): ASD TD $(n=14,307)^{43}$, and epilepsy $(n=44,889)^{44}$. In total, we identified 6 trait pairs with significant
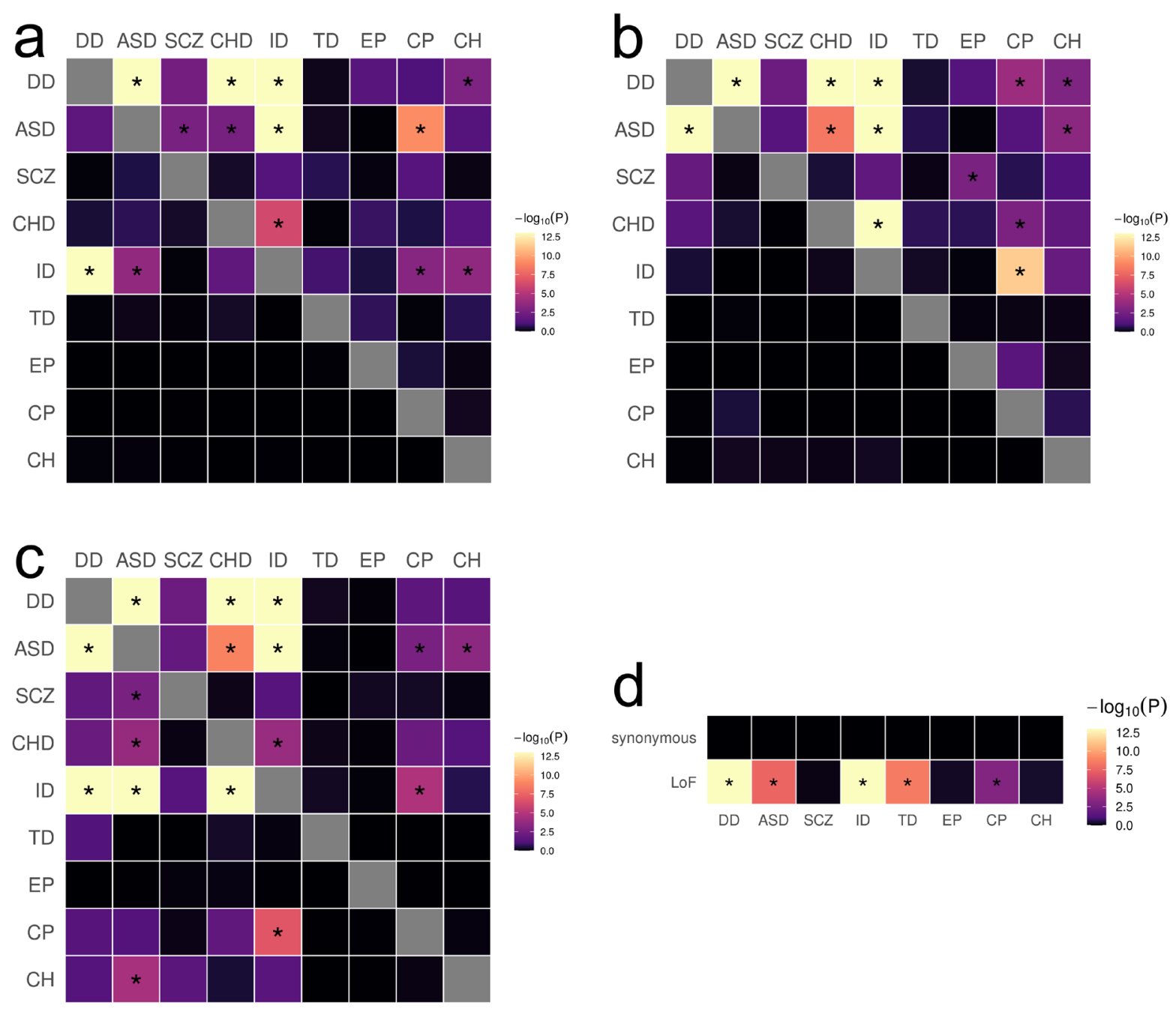

Figure 6. DNM enrichment correlations in disease-relevant gene sets. (a) Enrichment correlations in High-pLI genes (upper triangle) and Low-pLI genes (lower triangle) for LoF variants. (b) Enrichment correlations in HBE genes (upper triangle) and LBE genes (lower triangle) for LoF variants. (c) Enrichment correlations in HHE genes (upper triangle) and LHE genes (lower triangle) for LoF variants. (d) Enrichment correlations in CHD-related pathways for LoF and synonymous variants. Significant correlations (FDR $<0.05)$ are marked by asterisks. Results with $-\log _{10} P>13$ are truncated to 13 for visualization purpose. 


\section{Partitioning DNM enrichment correlation by gene set}

To gain biological insights into the shared genetic architecture of nine disorders, we repeated EncoreDNM correlation analysis in several gene sets. First, we defined genes with high/low probability of intolerance to LoF variants using $\mathrm{pLI}$ scores ${ }^{45}$, and identified genes with high/low brain expression (HBE/LBE) ${ }^{46}$ (Methods; Supplementary Table 10). We identified 11 and 12 disorder pairs showing significant enrichment correlations for LoF DNMs in high-pLI genes and HBE genes, respectively (Figure 6a-b). We observed fewer significant correlations for Dmis and Tmis variants in these gene sets (Supplementary Figures 12-13). All identified significant correlations were positive (Supplementary Tables 11-12). No significant correlations were identified for synonymous variants (Supplementary Figures 12-13) or between disorders and controls (Supplementary Figures 14-15).

We observed a clear enrichment of significant correlations in disease-relevant gene sets. Overall, high-pLI genes showed substantially stronger correlations across disorders than genes with low $\mathrm{pLI}$ (one-sided Kolmogorov-Smirnov test; $p=2.3 e-6$ ). Similarly, enrichment correlations were stronger in HBE genes than in LBE genes $(p=8.8 \mathrm{e}-7)$. Among the 11 disorder pairs showing significant enrichment correlations in high-pLI genes, two pairs, i.e., ASD-SCZ ( $\rho=0.68, p=2.4 \mathrm{e}-$ 3 ) and DD-CH ( $\rho=0.43, p=1.5 e-3)$, were not identified in the exome-wide analysis. We also identified four novel disorder pairs with significant correlations in HBE genes, including DD-CP $(\rho=0.80, \mathrm{p}=9.5 \mathrm{e}-5), \mathrm{DD}-\mathrm{CH}(\rho=0.67, \mathrm{p}=1.4 \mathrm{e}-3), \mathrm{ASD}-\mathrm{CH}(\rho=0.82, \mathrm{p}=4.7 \mathrm{e}-4)$, and SCZ-EP $(\rho=0.66, \mathrm{p}=2.0 \mathrm{e}-3)$. These novel enrichment correlations are consistent with known comorbidities between these disorders ${ }^{47,48}$ and findings based on significant risk genes $8,28,49,50$.

Furthermore, we estimated DNM enrichment correlations in genes with high/low expression in mouse developing heart (HHE/LHE) $)^{7}$ (Methods; Supplementary Table 10). We identified 9 significant enrichment correlations for LoF variants in HHE genes (Figure 6c). Strength of enrichment correlations did not show a significant difference between HHE and LHE genes $(p=0.846)$, possibly due to a lack of cardiac disorders in our analysis. Finally, we estimated enrichment correlations between $\mathrm{CHD}$ and other disorders in known pathways for $\mathrm{CHD}^{51}$ (Methods; Supplementary Table 10). We identified 5 significant correlations for LoF variants (Figure 6d), including a novel correlation between CHD and TD $(\rho=0.93, p=3.3 e-9)$. Of note, arrhythmia caused by $\mathrm{CHD}$ is a known risk factor for $T^{52}$. In these analyses, all significant enrichment correlations were positive (Supplementary Tables 13-14) and other variant classes showed generally weaker correlations than LoF variants (Supplementary Figures 16-17). We did not observe significant correlations in these gene sets between disorders and controls (Supplementary Figures 18-19). 


\section{Discussion}

In this paper, we introduced EncoreDNM, a novel statistical framework to quantify correlated DNM enrichment between two disorders. Through extensive simulations and analyses of DNM data for nine disorders, we demonstrated that our proposed mixed-effects Poisson regression model provides unbiased parameter estimates, shows well-controlled type-l error, and is robust to exome-wide technical biases. Leveraging exome-wide DNM counts and genomic context-based mutability data, EncoreDNM achieves superior fit for real DNM datasets compared to simpler models and provides statistically powerful and computationally efficient estimation of DNM enrichment correlation. Further, EncoreDNM can quantify concordant genetic effects for userdefined variant classes within pre-specified gene sets, thus is suitable for exploring diverse types of hypotheses and can provide crucial biological insights into the shared genetic etiology in multiple disorders.

Multi-trait analyses of GWAS data have revealed shared genetic architecture among many neuropsychiatric traits ${ }^{22,53,54}$. These findings have led to the identification of pleiotropic variants, genes, and hub genomic regions underlying many traits and have revealed multiple psychopathological factors jointly affecting human neurological phenotypes ${ }^{55,56}$. Although emerging evidence suggests that causal DNMs underlying several disorders with well-powered studies (e.g., CHD and neurodevelopmental disorders ${ }^{7}$ ) may be shared, our understanding of the extent and the mechanism underlying such sharing remains incomplete. Applied to DNM data for nine disorders, EncoreDNM identified pervasive enrichment correlations of DNMs. We observed particularly strong correlations in pathogenic variant classes (e.g., LoF and Dmis variants) and disease-relevant genes (e.g., genes with high pLI and genes highly expressed in relevant tissues). Genes underlying these correlations were significantly enriched in pathways involved in chromatin organization and modification and gene expression regulation. The DNM correlations were substantially attenuated in genes with lower expression and genes with frequent occurrences of LoF variants in the population. A similar attenuation was observed in less pathogenic variant classes (e.g., synonymous variants). Further, no significant correlations were identified between any disorder and healthy controls. These results lay the groundwork for future investigations of pleiotropic mechanisms of DNMs.

Our study has some limitations. First, a main goal in DNM research is to identify disease risk genes. EncoreDNM leverages exome-wide DNM counts to quantify shared genetic basis in multiple disorders but does not improve the analysis of gene-disease associations. Second, EncoreDNM assumes probands from different input studies to be independent. In rare cases when two studies have overlapping proband samples, enrichment correlation estimates may be inflated and must be interpreted with caution. Finally, genetic correlation methods based on GWAS summary data provided key motivations for the mixed-effects Poisson regression model in our study. Built upon genetic correlations, a plethora of methods have been developed in the 
GWAS literature to jointly model more than two GWAS ${ }^{57}$, identify and quantify common factors underlying multiple traits ${ }^{58,59}$, estimate causal effects among different traits ${ }^{60}$, and identify pleiotropic genomic regions through hypothesis-free scans ${ }^{23}$. Future directions of EncoreDNM include using enrichment correlation to improve gene discovery, learning the directional effects and the causal structure underlying multiple disorders, and dynamically searching for gene sets and annotation classes with shared genetic effects without pre-specifying the hypothesis.

Taken together, we provide a new analytic approach to an important problem in DNM studies. We believe EncoreDNM improves the statistical rigor in multi-disorder DNM modeling and opens up many interesting future directions in both method development and follow-up analyses in WES studies. As trio sample size in WES studies continues to grow, EncoreDNM will have broad applications and can greatly benefit DNM research.

\section{Methods}

\section{Statistical Model}

For a single study, we assume that DNM counts in a given variant class (e.g., synonymous variants) follow a mixed-effects Poisson model:

where $Y_{i}$ is the DNM count in the $i$-th gene, $N$ is the number of trios, $m_{i}$ is the de novo mutabilityfor the $i$-th gene (i.e., mutation rate per chromosome per generation) which is known a priori $^{26}$ (Supplementary Table 15), and $G$ is the total number of genes in the study. The elevation parameter $\beta$ quantifies the global elevation of mutation rate compared to mutability estimates based on genomic sequence alone. Gene-specific deviation from expected DNM rate is quantified by random effect $\phi_{i}$ with a dispersion parameter $\sigma$. Here, the $\phi_{i}$ are assumed to be independent across different genes, in which case the observed DNM counts of different genes are independent. We assume DNM counts in a given variant class for two diseases follow:

$$
\begin{gathered}
\log \left(\lambda_{i}\right)=\beta+\log \left(2 N m_{i}\right)+\phi_{i}, \\
\phi_{i} \sim \mathrm{N}\left(0, \sigma^{2}\right), \quad \text { for } i=1, \ldots, G,
\end{gathered}
$$

$$
\begin{gathered}
{\left[\begin{array}{l}
Y_{i 1} \\
Y_{i 2}
\end{array}\right] \sim \operatorname{Poisson}\left(\left[\begin{array}{l}
\lambda_{i 1} \\
\lambda_{i 2}
\end{array}\right]\right),} \\
\log \left(\left[\begin{array}{l}
\lambda_{i 1} \\
\lambda_{i 2}
\end{array}\right]\right)=\left[\begin{array}{l}
\beta_{1} \\
\beta_{2}
\end{array}\right]+\log \left(\left[\begin{array}{l}
2 N_{1} m_{i} \\
2 N_{2} m_{i}
\end{array}\right]\right)+\left[\begin{array}{l}
\phi_{i 1} \\
\phi_{i 2}
\end{array}\right], \\
{\left[\begin{array}{l}
\phi_{i 1} \\
\phi_{i 2}
\end{array}\right] \sim \operatorname{MVN}\left(\left[\begin{array}{l}
0 \\
0
\end{array}\right],\left[\begin{array}{cc}
\sigma_{1}^{2} & \rho \sigma_{1} \sigma_{2} \\
\rho \sigma_{1} \sigma_{2} & \sigma_{2}^{2}
\end{array}\right]\right),}
\end{gathered}
$$


where $Y_{i 1}, Y_{i 2}$ are the DNM counts for the $i$-th gene and $N_{1}, N_{2}$ are the trio sizes in two studies, respectively. Similar to the single-trait model, $m_{i}$ is the mutability for the $i$-th gene. $\beta_{1}, \beta_{2}$ are the elevation parameters, and $\phi_{i 1}, \phi_{i 2}$ are the gene-specific random effects with dispersion parameters $\sigma_{1}, \sigma_{2}$, for two disorders respectively. $\rho$ is the enrichment correlation which quantifies the concordance of the gene-specific DNM burden between two disorders. Here, $\beta_{1}, \beta_{2}, \sigma_{1}, \sigma_{2}, \rho$ are unknown parameters. The gene specific effects for two disorders are assumed to be independent for different genes. We also assume that there is no shared sample for two disorders, in which case $Y_{i 1}$ is independent with $Y_{i 2}$ given $\left[\begin{array}{l}\lambda_{i 1} \\ \lambda_{i 2}\end{array}\right]$.

\section{Parameter estimation}

We implement an MLE procedure to estimate unknown parameters. For single-trait analysis, the log-likelihood function can be expressed as follows:

$$
l(\beta, \sigma \mid \boldsymbol{Y})=\sum_{i=1}^{G} \log \left[\int \exp \left(-\lambda_{i}\right) \lambda_{i}^{Y i} * f\left(\phi_{i}\right) d \phi_{i}\right]+C,
$$

where $\quad \boldsymbol{Y}=\left[Y_{1}, \ldots, Y_{G}\right]^{T}, \quad \lambda_{i}=2 N m_{i} \exp \left(\beta+\phi_{i}\right) \quad, \quad C=-\sum_{i=1}^{G} \log \left(Y_{i} !\right) \quad, \quad$ and $\quad f\left(\phi_{i}\right)=$ $\frac{1}{\sqrt{2 \pi} \sigma} \exp \left(-\frac{\phi_{i}^{2}}{2 \sigma^{2}}\right)$. Note that there is no closed form for the integral in the log-likelihood function. Therefore, we use Monte Carlo integration to evaluate the log-likelihood function. Let $\phi_{i j}=\sigma \xi_{i j}$, where the $\xi_{i j}$ are independently and identically distributed random variables following a standard normal distribution. We have

$$
l(\beta, \sigma \mid \boldsymbol{Y}) \approx l^{\prime}(\beta, \sigma \mid \boldsymbol{Y})=\sum_{i=1}^{G} \log \left[\sum_{j=1}^{M} \exp \left(-\lambda_{i j}\right) \lambda_{i j}^{Y_{i}}\right]+C
$$

where $\lambda_{i j}=2 N m_{i} \exp \left(\beta+\sigma \xi_{i j}\right)$, and $M$ is the Monte Carlo sample size which is set to be 1000 . Then, we could obtain the MLE of $\beta, \sigma$ through maximization of $l^{\prime}(\beta, \sigma \mid \boldsymbol{Y})$. We obtain the standard error of the MLE through inversion of the observed Fisher information matrix.

The estimation procedure can be generalized to multi-trait analysis. Log-likelihood function can be expressed as follows:

$$
l\left(\beta_{1}, \beta_{2}, \sigma_{1}, \sigma_{2}, \rho \mid \boldsymbol{Y}_{1}, \boldsymbol{Y}_{2}\right)=\sum_{i=1}^{G} \log \left[\int \exp \left(-\lambda_{i 1}-\lambda_{i 2}\right) \lambda_{i 1}^{Y_{i 1}} \lambda_{i 2}^{Y i 2} * f\left(\phi_{i 1}, \phi_{i 2}\right) d \phi_{i 1} d \phi_{i 2}\right]+C,
$$

where $\boldsymbol{Y}_{1}=\left[Y_{11}, \ldots, Y_{G 1}\right]^{T}, \boldsymbol{Y}_{2}=\left[Y_{12}, \ldots, Y_{G 2}\right]^{T}, \lambda_{i 1}=2 N_{1} m_{i} \exp \left(\beta_{1}+\phi_{i 1}\right), \lambda_{i 2}=2 N_{2} m_{i} \exp \left(\beta_{2}+\right.$ $\left.\phi_{i 2}\right), \quad C=-\sum_{i=1}^{G}\left[\log \left(Y_{i 1} !\right)+\log \left(Y_{i 2} !\right)\right]$, and $f\left(\phi_{i 1}, \phi_{i 2}\right)=\frac{1}{2 \pi \sigma_{1} \sigma_{2} \sqrt{1-\rho^{2}}} \exp \left[-\frac{1}{2 \sqrt{1-\rho^{2}}}\left(\frac{\phi_{i 1}^{2}}{\sigma_{1}^{2}}+\frac{\phi_{i 2}^{2}}{\sigma_{2}^{2}}-\right.\right.$ $\left.\left.\frac{2 \rho \phi_{i 1} \phi_{i 2}}{\sigma_{1} \sigma_{2}}\right)\right]$. We use Monte Carlo integration to evaluate the log-likelihood function. Let $\phi_{i 1 j}=$ 
$439 \sigma_{1} \xi_{i 1 j}$ and $\phi_{i 2 j}=\sigma_{2}\left(\rho \xi_{i 1 j}+\sqrt{1-\rho^{2}} \xi_{i 2 j}\right)$, where the $\xi_{i 1 j}$ and $\xi_{i 2 j}$ are independently and

440 identically distributed random variables following a standard normal distribution. We have

$$
l\left(\beta_{1}, \beta_{2}, \sigma_{1}, \sigma_{2}, \rho \mid \boldsymbol{Y}_{1}, \boldsymbol{Y}_{2}\right) \approx l^{\prime}\left(\beta_{1}, \beta_{2}, \sigma_{1}, \sigma_{2}, \rho \mid \boldsymbol{Y}_{1}, \boldsymbol{Y}_{2}\right)=\sum_{i=1}^{G} \log \left[\sum_{j=1}^{M} \exp \left(-\lambda_{i 1 j}-\lambda_{i 2 j}\right) \lambda_{i 1 j}^{Y_{i 1}} \lambda_{i 2 j}^{Y_{i 2}}\right]+C,
$$

where $\lambda_{i 1 j}=2 N_{1} m_{i} \exp \left(\beta_{1}+\sigma_{1} \xi_{i 1 j}\right)$ and $\lambda_{i 2 j}=2 N_{2} m_{i} \exp \left[\beta_{2}+\sigma_{2}\left(\rho \xi_{i 1 j}+\sqrt{1-\rho^{2}} \xi_{i 2 j}\right)\right]$. Then, we obtain the MLE of $\beta_{1}, \beta_{2}, \sigma_{1}, \sigma_{2}, \rho$ through maximization of $l^{\prime}\left(\beta_{1}, \beta_{2}, \sigma_{1}, \sigma_{2}, \rho \mid \boldsymbol{Y}_{1}, \boldsymbol{Y}_{2}\right)$. Standard error of MLE can be obtained through inversion of the observed Fisher information matrix.

\section{DNM data and variant annotation} the original release ${ }^{13}$ were not in an editable format and were instead collected from denovo- $\mathrm{db}^{61}$. We used ANNOVAR ${ }^{62}$ to annotate all DNMs. Synonymous variants were determined based on the 'synonymous SNV' annotation in ANNOVAR; Variants with 'startloss', 'stopgain', 'stoploss', 'splicing', 'frameshift insertion', 'frameshift deletion', or 'frameshift substitution' annotations were classified as LoF; Dmis variants were defined as nonsynonymous SNVs predicted to be deleterious by MetaSVM ${ }^{63}$; nonsynonymous SNVs predicted to be tolerable by MetaSVM were classified as Tmis. Other DNMs which did not fall into these categories were removed from the analysis. For each variant class, we estimated the mutability of each gene using a sequencebased mutation mode ${ }^{26}$ while adjusting for the sequencing coverage factor based on control trios as previously described ${ }^{12}$ (Supplementary Table 15). We included 18,454 autosomal proteincoding genes in our analysis. TTN was removed due to its substantially larger size.

\section{Implementation of mTADA}

The software mTADA requires the following parameters as inputs: proportion of risk genes $\left(\pi_{1}^{S}, \pi_{2}^{S}\right)$, mean relative risks $\left(\bar{\gamma}_{1}^{S}, \bar{\gamma}_{2}^{S}\right)$, and dispersion parameters $\left(\bar{\beta}_{1}^{S}, \bar{\beta}_{2}^{S}\right)$ for both disorders. We used extTADA $^{10}$ to estimate these parameters as suggested by the mTADA paper ${ }^{9}$. mTADA reported the estimated proportion of shared risk genes $\pi_{3}$ (posterior mode of $\pi_{3}$ ) and its corresponding 95\% credible interval [LB, UB]. We considered $\pi_{1}^{S} * \pi_{2}^{S}$ as the expected proportion of shared risk genes, and there is significant genetic sharing between two disorders when LB $>\pi_{1}^{S} * \pi_{2}^{S}$. Pvalue for $\pi_{3}$ was calculated by comparing $\pi_{1}^{S} * \pi_{2}^{S}$ to the posterior distribution of $\pi_{3}$. Number of MCMC chain was set as 2 and number of iterations was set as 10,000.

\section{Simulation settings}

We assessed the performance of EncoreDNM under the mixed-effects Poisson model. We 
476 the smallest median mutability values across all genes. First, we performed single-trait simulations to assess estimation precision of elevation parameter $\beta$ and dispersion parameter $\sigma$. We set the true values of $\beta$ to be $-0.5,-0.25$, and 0 , and the true values of $\sigma$ to be $0.5,0.75$, and 1. These values were chosen based on the estimated parameters in real DNM data analyses and ensured simulation settings to be realistic. Next, we performed simulations for cross-trait analysis to assess estimation precision of enrichment correlation $\rho$, whose true values were set to be $0,0.2,0.4,0.6$, and 0.8 . Sample size for each disorder was set to be 5,000 . Coverage rate was calculated as the percentage of simulations that the $95 \%$ Wald confidence interval covered the true parameter value. Each parameter setting was repeated 100 times.

We also carried out simulations to compare the performance of EncoreDNM and mTADA. Type I error and statistical power for EncoreDNM were calculated as the proportion of simulation repeats that $\mathrm{p}$-value for enrichment correlation $\rho$ was smaller than 0.05 . and the proportion of simulation repeats that $p$-value for estimated proportion of shared risk genes $\pi_{3}$ was smaller than 0.05 was used for mTADA. We aggregated all variant classes together, so mutability for each gene was determined as the sum of mutabilities across four variant classes (i.e. LoF, Dmis, Tmis, and synonymous).

First, we simulated DNM data under the mixed-effects Poisson model. To see whether two methods would produce false positive findings, we performed simulations under the null hypothesis that the enrichment correlation $\rho$ is zero. We compared two methods under a range of parameter combinations of $(\beta, \sigma, N)$ for both disorders: $(-0.25,0.75,5000)$ for the baseline setting, $(-1,0.75,5000)$ for a setting with small $\beta,(-0.25,0.5,5000)$ for a setting with small $\sigma$, and $(-0.25,0.75,1000)$ for a setting with small sample size. We also assessed the statistical power of two methods under the alternative hypothesis. True value of enrichment correlation $\rho$ was set to be $0.05,0.1,0.15$, and 0.2 . In the power analysis, parameters $(\beta, \sigma, N)$ were fixed at ($0.25,0.75,5000)$ as in the baseline setting when both methods had well-controlled type-I error.

To ensure a fair comparison, we also compared EncoreDNM and mTADA under a multinomial model, which is different from the data generation processes for the two approaches. For each disorder $(k=1,2)$, we randomly selected causal genes of proportion $\pi_{k}^{S}$. A proportion (i.e., $\left.\pi_{3}\right)$ of causal genes overlap between two disorders. We assumed that the total DNM count to follow a Poisson distribution: $C_{k} \sim$ Poisson $\left(u_{k} * 2 N_{k} \sum_{i=1}^{G} m_{i}\right)$, where $u_{k}$ represents an elevation factor to represent systematic bias in the data. Let $\mathbf{Y}_{k}$ denote the vector of DNMs counts in the exome, $\boldsymbol{m}$ denote the vector of mutability values for all genes, and $\boldsymbol{m}_{\text {causal,k }}$ denote the vector of

511 mutability with values set to be 0 for non-causal genes of disorder $k$. We assumed that a 512 proportion $p_{k}$ of the probands could be attributed to DNMs burden in causal genes, and $1-p_{k}$ 513 of the probands obtained DNMs by chance:

$$
\begin{gathered}
\mathbf{Y}_{k}=\mathbf{Y}_{\text {causal }, k}+\mathbf{Y}_{\text {background }, k}, \\
\mathbf{Y}_{\text {causal }, k} \sim \operatorname{Multinomial}\left(p_{k} C_{k}, \boldsymbol{m}_{\text {causal }, k}\right), \\
\mathbf{Y}_{\text {background }, k} \sim \operatorname{Multinomial}\left(\left(1-p_{k}\right) C_{k}, \boldsymbol{m}\right) .
\end{gathered}
$$


517 To check whether false positive findings could arise, we performed simulations under the null

518 hypothesis that $\pi_{3}=\pi_{1}^{S} * \pi_{2}^{S}$ across a range of parameter combinations of $\left(u, p, N, \pi^{S}\right)$ for both 519 disorders: $(0.95,0.25,5000,0.1)$ for the baseline setting, $(0.75,0.25,5000,0.1)$ for a setting with 520 small $u$ (i.e., reduced total mutation count), $(0.95,0.15,5000,0.1)$ for a setting with small $p$ 521 (fewer probands explained by DNMs), and $(0.95,0.25,1000,0.1)$ for a setting with smaller sample 522 size. We also assessed the statistical power of two methods under the alternative hypothesis that $523 \pi_{3}>\pi_{1}^{S} * \pi_{2}^{S}$. In power analysis, $\left(u, p, N, \pi^{S}\right)$ were fixed at $(0.95,0.25,5000,0.1)$ as in the baseline 524 setting when type-l error for both methods were well-calibrated.

525

\section{Comparison to the fixed-effects Poisson model}

527

For single-trait analysis, the fixed-effects Poisson model assumes that

$$
\log \left(\lambda_{i}\right)=\beta+\log \left(2 N m_{i}\right), \quad \text { for } i=1, \ldots, G \text {. }
$$

Note that the fixed-effects Poisson model is a special case of our proposed mixed-effects Poisson model when $\sigma=0$. We compared the two models using likelihood ratio test. Under the null log likelihood of the fitted mixed-effects and fixed-effects Poisson models respectively.

\section{Recurrent genes and DNMs}

We used FUMA ${ }^{64}$ to perform GO enrichment analysis for genes harboring LoF DNMs in multiple disorders. Due to potential sample overlap between the studies of $\mathrm{DD}^{3}$ and $\mathrm{ID}^{2}$, we excluded ID from the analysis of recurrent DNMs. We calculated the probability of observing two identical DNMs in two disorders using a Monte Carlo simulation method. For each disorder, we simulated exome-wide DNMs profile from a multinomial distribution, where the size was fixed at the observed DNM count and the per-base mutation probability was determined by the tri-nucleotide base context. We repeated the simulation procedure 100,000 times to evaluate the significance of recurrent DNMs. Lollipop plots for recurrent mutations were generated using MutationMapper on the cBio Cancer Genomics Portal ${ }^{65}$.

\section{Implementation of cross-trait LD score regression}

We used cross-trait $\operatorname{LDSC}^{18}$ to estimate genetic correlations between disorders. LD scores were HapMap 3 SNPs were used as observations in the explanatory variable with the --merge-alleles flag. Intercepts were not constrained in the analyses.

\section{Estimating enrichment correlation in gene sets}


557 Genes with a high/low probability of intolerance to LoF variants (high-pLI/low-pLI) were defined

558 as the 4,614 genes in the upper/lower quartiles of $\mathrm{pLI}$ scores ${ }^{45}$. Genes with high/low brain 559 expression (HBE/LBE) were defined as the 4,614 genes in the upper/lower quartiles of expression 560 in the human fetal brain ${ }^{46}$. Genes with high/low heart expression (HHE/LHE) were defined as the 5614,614 genes in the upper/lower quartiles of expression in the developing heart of embryonic 562 mouse $^{67}$. Five biological pathways have been reported to be involved in CHD: chromatin 563 remodeling, Notch signaling, cilia function, sarcomere structure and function, and RAS signaling ${ }^{51}$. 564 We extracted 1730 unique genes that belong to these five pathways from the gene ontology 565 database ${ }^{68}$ and referred to the union set as CHD-related genes. We repeated EncoreDNM 566 enrichment correlation analysis in these gene sets. One-sided Kolmogorov-Smirnov test was 567 used to assess the statistical difference between enrichment correlation signal strength in different 568 gene sets.

569

URLs

571

572 GWAS summary statistics data of ASD, SCZ, and TD were downloaded on the PGC website, 573 https://www.med.unc.edu/pgc/download-results/; Summary statistics of cognitive performance 574 were downloaded on the SSGAC website, https://www.thessgac.org/data; Summary statistics of 575 epilepsy were downloaded on the epiGAD website, http://www.epigad.org/; pLI scores were 576 downloaded from gnomAD v3.1 repository https://gnomad.broadinstitute.org/downloads; mTADA, 577 https://github.com/hoangtn/mTADA; denovo-db, https://denovo-db.gs.washington.edu/denovo$578 \mathrm{db} /$; MutationMapper on cBioPortal, https://www.cbioportal.org/mutation mapper; LDSC, $579 \quad$ https://github.com/bulik/ldsc.

580

581

582

Code availability

583

584

EncoreDNM software is available at https://github.com/ghm17/EncoreDNM. 


\section{Acknowledgements}

587 LH acknowledges research support from the National Science Foundation of China (Grant No.

588 12071243) and Shanghai Municipal Science and Technology Major Project (Grant No.

589 2017SHZDZX01). QL acknowledges research support from the University of Wisconsin-Madison

590 Office of the Chancellor and the Vice Chancellor for Research and Graduate Education with

591 funding from the Wisconsin Alumni Research Foundation and the Waisman Center pilot grant

592 program at University of Wisconsin-Madison. $\mathrm{HZ}$ acknowledges research support from the

593 National Institutes of Health (Grant No. R03HD100883)

594

\section{Author contribution}

596 H.G., L.H., and Q.L. designed the study.

597 H.G. performed data analysis and implemented the software.

598 Y.S. implemented an early version of the method.

599 S.C.J., X.Z., and B.L. assisted DNM and mutability data preparation.

600 R.P.L and M.B. advised on disease biology, data interpretation, and genetic issues.

601 H.Z. and Q.L. advised on statistical issues.

602 H.G., L.H., and Q.L. wrote the manuscript.

603 All authors contributed in manuscript editing and approved the manuscript.

604

605 Competing financial interests

606 The authors declare no competing financial interests. 


\section{References}

609 1. Veltman, J.A. \& Brunner, H.G. De novo mutations in human genetic disease. Nature Reviews Genetics 13, 565-575 (2012).

6112 2. Lelieveld, S.H. et al. Meta-analysis of 2,104 trios provides support for 10 new genes for intellectual disability. Nature neuroscience 19, 1194-1196 (2016).

3. Kaplanis, J. et al. Evidence for 28 genetic disorders discovered by combining healthcare and research data. Nature 586, 757-762 (2020).

4. Satterstrom, F.K. et al. Large-scale exome sequencing study implicates both developmental and functional changes in the neurobiology of autism. Cell 180, 568-584. e23 (2020).

5. Hoischen, A., Krumm, N. \& Eichler, E.E. Prioritization of neurodevelopmental disease genes by discovery of new mutations. Nature neuroscience 17, 764 (2014).

6. $\quad$ Fromer, M. et al. De novo mutations in schizophrenia implicate synaptic networks. Nature 506, 179184 (2014).

7. Homsy, J. et al. De novo mutations in congenital heart disease with neurodevelopmental and other congenital anomalies. Science 350, 1262-1266 (2015).

8. $\mathrm{Li}, \mathrm{J}$. et al. Genes with de novo mutations are shared by four neuropsychiatric disorders discovered from NPdenovo database. Molecular psychiatry 21, 290-297 (2016).

9. Nguyen, T.-H. et al. mTADA is a framework for identifying risk genes from de novo mutations in multiple traits. Nature Communications 11, 2929 (2020).

10. Nguyen, H.T. et al. Integrated Bayesian analysis of rare exonic variants to identify risk genes for schizophrenia and neurodevelopmental disorders. Genome medicine 9, 114 (2017).

11. Willsey, A.J. et al. The psychiatric cell map initiative: a convergent systems biological approach to illuminating key molecular pathways in neuropsychiatric disorders. Cell 174, 505-520 (2018).

12. Jin, S.C. et al. Contribution of rare inherited and de novo variants in 2,871 congenital heart disease probands. Nature genetics 49, 1593 (2017).

13. Allen, A.S. et al. De novo mutations in epileptic encephalopathies. Nature 501, 217-221 (2013).

14. Jin, S.C. et al. Mutations disrupting neuritogenesis genes confer risk for cerebral palsy. Nature Genetics 52, 1046-1056 (2020).

15. Howrigan, D.P. et al. Exome sequencing in schizophrenia-affected parent-offspring trios reveals risk conferred by protein-coding de novo mutations. Nature Neuroscience 23, 185-193 (2020).

16. Zhang, Y. et al. Comparison of methods for estimating genetic correlation between complex traits using GWAS summary statistics. Briefings in bioinformatics (2021).

18. Bulik-Sullivan, B. et al. An atlas of genetic correlations across human diseases and traits. Nature genetics 47, 1236 (2015).

645 19. Lu, Q. et al. A Powerful Approach to Estimating Annotation-Stratified Genetic Covariance via GWAS Summary Statistics. Am J Hum Genet 101, 939-964 (2017).

647 20. Ning, Z., Pawitan, Y. \& Shen, X. High-definition likelihood inference of genetic correlations across 
human complex traits. Nature genetics 52, 859-864 (2020).

21. Shi, H., Mancuso, N., Spendlove, S. \& Pasaniuc, B. Local genetic correlation gives insights into the shared genetic architecture of complex traits. The American Journal of Human Genetics 101, 737751 (2017).

22. Brainstorm, C. et al. Analysis of shared heritability in common disorders of the brain. Science 360(2018).

23. Guo, H., Li, J.J., Lu, Q. \& Hou, L. Detecting local genetic correlations with scan statistics. Nature Communications 12, 2033 (2021).

24. Zhang, Y. et al. SUPERGNOVA: local genetic correlation analysis reveals heterogeneous etiologic sharing of complex traits. Genome biology 22, 1-30 (2021).

25. Wei, Q. et al. A Bayesian framework for de novo mutation calling in parents-offspring trios. Bioinformatics 31, 1375-1381 (2015).

26. Samocha, K.E. et al. A framework for the interpretation of de novo mutation in human disease. Nature genetics 46, 944-950 (2014).

27. Willsey, A.J. et al. De novo coding variants are strongly associated with Tourette disorder. Neuron 94, 486-499. e9 (2017).

28. Jin, S.C. et al. Exome sequencing implicates genetic disruption of prenatal neuro-gliogenesis in sporadic congenital hydrocephalus. Nature medicine 26, 1754-1765 (2020).

29. Krumm, N. et al. Excess of rare, inherited truncating mutations in autism. Nature genetics 47, 582588 (2015).

30. Lek, M. et al. Analysis of protein-coding genetic variation in 60,706 humans. Nature 536, 285-291 (2016).

31. Hormozdiari, F., Penn, O., Borenstein, E. \& Eichler, E.E. The discovery of integrated gene networks for autism and related disorders. Genome research 25, 142-154 (2015).

32. Christensen, D. et al. Prevalence of cerebral palsy, co-occurring autism spectrum disorders, and motor functioning-A utism and D evelopmental D isabilities M onitoring N etwork, USA, 2008. Developmental Medicine \& Child Neurology 56, 59-65 (2014).

33. Reid, S.M., Meehan, E.M., Arnup, S.J. \& Reddihough, D.S. Intellectual disability in cerebral palsy: a population-based retrospective study. Developmental Medicine \& Child Neurology 60, 687-694 (2018).

34. Garne, E. et al. Cerebral palsy and congenital malformations. European Journal of Paediatric Neurology 12, 82-88 (2008).

35. Lumenta, C.B. \& Skotarczak, U. Long-term follow-up in 233 patients with congenital hydrocephalus. Child's Nervous System 11, 173-175 (1995).

36. O'Roak, B.J. et al. Sporadic autism exomes reveal a highly interconnected protein network of de novo mutations. Nature 485, 246-250 (2012).

37. Cardozo, T. \& Pagano, M. The SCF ubiquitin ligase: insights into a molecular machine. Nature reviews Molecular cell biology 5, 739-751 (2004).

38. Jansen, S. et al. De novo variants in FBXO11 cause a syndromic form of intellectual disability with 
behavioral problems and dysmorphisms. European Journal of Human Genetics 27, 738-746 (2019).

39. Gregor, A. et al. De novo variants in the F-box protein FBXO11 in 20 individuals with a variable neurodevelopmental disorder. The American Journal of Human Genetics 103, 305-316 (2018).

40. Grove, J. et al. Identification of common genetic risk variants for autism spectrum disorder. Nature genetics 51, 431 (2019).

41. Ripke, S., Walters, J.T., O'Donovan, M.C. \& Consortium, S.W.G.o.t.P.G. Mapping genomic loci prioritises genes and implicates synaptic biology in schizophrenia. MedRxiv (2020).

42. Lee, J.J. et al. Gene discovery and polygenic prediction from a genome-wide association study of educational attainment in 1.1 million individuals. Nature genetics 50, 1112-1121 (2018).

43. Yu, D. et al. Interrogating the genetic determinants of Tourette's syndrome and other tic disorders through genome-wide association studies. American Journal of Psychiatry 176, 217-227 (2019).

44. Abou-Khalil, B. et al. Genome-wide mega-analysis identifies 16 loci and highlights diverse biological mechanisms in the common epilepsies. Nature Communications 9, 5269 (2018).

45. Karczewski, K.J. et al. The mutational constraint spectrum quantified from variation in 141,456 humans. Nature 581, 434-443 (2020).

702

46. Werling, D.M. et al. Whole-genome and RNA sequencing reveal variation and transcriptomic

703 coordination in the developing human prefrontal cortex. Cell reports 31, 107489 (2020).

705

47. Kielinen, M., Rantala, H., Timonen, E., Linna, S.-L. \& Moilanen, I. Associated medical disorders

48. Kilincaslan, A. \& Mukaddes, N.M. Pervasive developmental disorders in individuals with cerebral palsy. Developmental Medicine \& Child Neurology 51, 289-294 (2009).

709

49. Kume, T. et al. The forkhead/winged helix gene Mf1 is disrupted in the pleiotropic mouse mutation

50. Cao, M. \& Wu, J.I. Camk2a-Cre-mediated conditional deletion of chromatin remodeler Brg1 causes perinatal hydrocephalus. Neuroscience letters 597, 71-76 (2015).

713

51. Zaidi, S. \& Brueckner, M. Genetics and genomics of congenital heart disease. Circulation research 120, 923-940 (2017).

52. Gulisano, M. et al. Cardiovascular safety of aripiprazole and pimozide in young patients with

53. Lee, S.H. et al. Genetic relationship between five psychiatric disorders estimated from genomewide SNPs. Nature genetics 45, 984 (2013).

54. Gratten, J., Wray, N.R., Keller, M.C. \& Visscher, P.M. Large-scale genomics unveils the genetic architecture of psychiatric disorders. Nature neuroscience 17, 782 (2014).

721

722 Lee, P.H. et al. Genomic relationships, novel loci, and pleiotropic mechanisms across eight psychiatric disorders. Cell 179, 1469-1482. e11 (2019).

56. Wang, Q., Yang, C., Gelernter, J. \& Zhao, H. Pervasive pleiotropy between psychiatric disorders and immune disorders revealed by integrative analysis of multiple GWAS. Human genetics 134, 1195-1209 (2015).

727 58. Grotzinger, A.D. et al. Genomic structural equation modelling provides insights into the multivariate 
genetic architecture of complex traits. Nature human behaviour 3, 513 (2019).

59. Grotzinger, A.D. et al. Genetic Architecture of 11 Major Psychiatric Disorders at Biobehavioral, Functional Genomic, and Molecular Genetic Levels of Analysis. medRxiv (2020).

60. Pickrell, J.K. et al. Detection and interpretation of shared genetic influences on 42 human traits. Nature genetics 48, 709 (2016).

61. Turner, T.N. et al. denovo-db: A compendium of human de novo variants. Nucleic acids research 45, D804-D811 (2017).

62. Wang, K., Li, M. \& Hakonarson, H. ANNOVAR: functional annotation of genetic variants from highthroughput sequencing data. Nucleic acids research 38, e164-e164 (2010).

63. Dong, C. et al. Comparison and integration of deleteriousness prediction methods for nonsynonymous SNVs in whole exome sequencing studies. Human molecular genetics 24, 21252137 (2015).

64. Watanabe, K., Taskesen, E., Van Bochoven, A. \& Posthuma, D. Functional mapping and annotation of genetic associations with FUMA. Nature communications 8, 1-11 (2017).

65. Cerami, E. et al. The cBio cancer genomics portal: an open platform for exploring multidimensional cancer genomics data. (AACR, 2012).

66. Consortium, G.P. A global reference for human genetic variation. Nature 526, 68-74 (2015).

67. Zaidi, S. et al. De novo mutations in histone-modifying genes in congenital heart disease. Nature

68. Ashburner, M. et al. Gene ontology: tool for the unification of biology. Nature genetics 25, 25-29 (2000). 